\title{
Avaliação do potencial de uso do deflectômetro de impacto leve para medidas de deflexão em pavimentos urbanos
}

\author{
Evaluation of the potential of use of the Portable Lightweight \\ Deflectometer (LFWD) to measure deflections in urban pavements
}

\section{Paulo Roberto Rodrigues ${ }^{1}$, Adriana Goulart dos Santos ${ }^{2}$, Edgar Odebrecht ${ }^{3}$}

\author{
${ }^{1}$ Secretaria de Infraestrutura Urbana, Prefeitura Municipal de Joinville, Santa Catarina - Brasil, paulo.rodrigues@joinville.sc.gov.br \\ 2Universidade do Estado de Santa Catarina, Santa Catarina-Brasil, adriana.santos@udesc.br \\ 3Universidade do Estado de Santa Catarina, Santa Catarina - Brasil, edgar@geoforma.com.br
}

\section{Recebido:}

3 de junho de 2019

Aceito para publicação:

20 de março de 2020

Publicado:

15 de dezembro de 2020

Editor de área:

Francisco Thiago Aragão

\section{Palavras-chaves:}

Deflectômetro de peso leve.

Deflexão.

Correlação.

Pavimento urbano.

\section{Keywords:}

Portable lightweight deflectometer.

Deflection.

Correlation.

Urban pavement.

DOI:10.14295/transportes.v28i5.2078

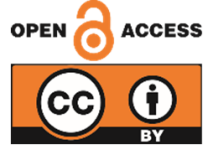

\begin{abstract}
RESUMO
O objetivo principal deste estudo foi avaliar o potencial de uso do Deflectômetro de Impacto Leve (LFWD) para medir deflexões em pavimentos urbanos. Para isso, avaliaram-se medidas de deflexões aferidas pelo LFWD, viga Benkelman (VB) e o ensaio de carga com placa (PL) em pavimentos construídos com diferentes materiais, espessuras de revestimento asfáltico e idades de operação. Determinaram-se modelos de regressão linear, que relacionaram as medidas de deflexão entre o LFWD e a VB, e o PL. Os resultados mostraram que não houveram boas relações lineares em estruturas compostas por camadas de revestimento asfáltico mais espessas, como também em estruturas com revestimento asfáltico sobre paralelepípedos. Houve boa relação linear em estruturas compostas por revestimento asfáltico mais delgado. Este estudo estimula o uso de equipamentos dinâmicos de ensaios não destrutivos para medição de deformações elásticas para o projeto de manutenção e reabilitação de vias de baixo volume de tráfego.
\end{abstract}

\section{ABSTRACT}

The main objective of this study was to available the potential of use of the Portable Lightweight Deflectometer (LFWD) to measure deflections in urban pavements. For this, measurements of deflections measured by the LFWD, benkelman beam (VB) and the plate load test (PL) on pavements constructed by different materials, layer thickness and different stages of its service life. Linear regression models were determined, which related the deflection measurements between LFWD and VB and PL. The results showed that there weren't good linear relations in structures with thicker bituminous layer, as well as in structures with bituminous layer on stone blocks. There were good linear relations in structures composed by thinner bituminous layer on granular base. This study stimulates the use of dynamic non-destructive testing equipment to measure elastic deformations for the design of maintenance and rehabilitation of low volume roads.

\section{INTRODUCÃO}

O sistema de transporte viário exerce um importante papel na sociedade, com influência direta em seu desenvolvimento econômico e social. 0 efeito das cargas dos veículos é a principal causa da deterioração do pavimento ao longo do tempo, seguido pela influência das condições meteorológicas e erros de projeto ou construção que podem intensificar esses defeitos, resultando em rápida diminuição da capacidade de carga do pavimento (Marecos et al., 2017). 
A avaliação do desempenho do pavimento ao longo do tempo de serviço é essencial. Para isso é necessário não só avaliar o seu desempenho quanto ao aspecto funcional, que se relaciona à apreciação da superfície do pavimento quanto ao conforto ao rolamento, mas também o quanto ao aspecto estrutural, o qual vai caracterizar a perda de capacidade suporte, acarretando em uma significativa redução de sua vida útil.

A avaliação estrutural do pavimento está associada ao conceito de capacidade de carga. Os defeitos estruturais dos pavimentos resultam, especialmente, da repetição das cargas veiculares e vinculam-se às deformações elásticas e plásticas. As deformações elásticas são mensuradas por equipamentos chamados de deflectômetros. Estes equipamentos medem os deslocamentos verticais, denominados de deflexão do pavimento. As deformações elásticas são responsáveis pelo surgimento da maioria dos trincamentos ao longo da vida do pavimento levando-o à ruptura por fadiga (Bernucci et al., 2007).

O projeto e execução de pavimentos objetivam que o mesmo suporte as ações das cargas dos veículos, que provocam carregamentos que geram estados de tensões durante a passagem do veículo em toda a estrutura. A magnitude dessas tensões depende da espessura das camadas e do módulo de elasticidade dos materiais que compõem a estrutura (Garcia e Castro, 2011). Embora a estrutura do pavimento seja projetada para resistir às solicitações de cargas dentro do período de projeto, esta estrutura sofre ruptura ou deformação excessiva quando as tensões excedem a capacidade dos materiais resistirem a tais esforços.

As medidas de deflexões podem ser efetuadas por métodos não destrutivos, conhecidos na literatura internacional como Nondestructive Deflection Testing - NDT. Os ensaios não destrutivos causam menos interferências no trânsito local que os métodos destrutivos, possibilitando assim maior facilidade para a avaliação estrutural do pavimento em qualquer período de sua vida de serviço, além de permitir que a área avaliada seja analisada por mais de um método (Lopes, 2010).

Os ensaios não destrutivos permitem obter bacias de deflexão para um determinado ponto de ensaio no pavimento, quando sobre o mesmo incide uma carga pontual ou rolante. Os equipamentos que utilizam a carga pontual para a leitura das deformações, medem a deflexão resultante ao carregamento de uma carga estática sobre um determinado ponto da superfície do pavimento. Por outro lado, os ensaios realizados por carregamentos de carga dinâmica utilizam equipamentos que, através de uma carga aplicada pela passagem de um eixo padrão, permite o traçado da deformada do pavimento, relativo a um ponto fixo. Este tipo de ensaio apresenta a vantagem de induzir nos pavimentos ações semelhantes às do tráfego em termos da grandeza das cargas aplicadas (Francisco, 2012).

Dentre os equipamentos de avaliação não destrutiva pode-se citar a Viga Benkelman (VB), que é um teste de campo para avaliação de deformações em pavimentos bastante conhecido pelos engenheiros rodoviários. Na década de 60 substituiu a prova de carga com placa circular, por ser um procedimento simples e mais ágil, cujo carregamento de carga estática é feito com os próprios pneus de um caminhão, ao invés da placa circular (Albernaz,1997).

Entretanto, devido aos custos logísticos e o tempo requerido na sua execução levaram os técnicos e pesquisadores da área de pavimentação à procura de soluções mais versáteis. Assim surgiu o equipamento Deflectômetro de Impacto Leve ou o Light Falling Weight Deflectometer (LFWD). O LFWD é um equipamento portátil, desenvolvido na Alemanha no início dos anos 80, que avalia materiais pela sua deformação, quando submetidos a uma carga dinâmica 
(Elhakim et al., 2014). O LFWD, assim como a VB, é utilizado para controle de qualidade das camadas do pavimento (subleito, sub-base e base), em pavimentos novos ou em obras já existentes (Nazaal, 2003).

O LFWD surgiu como alternativa ao equipamento Falling Weight Deflectometer (FWD) convencional, especialmente em situações onde se possam encontrar dificuldades de acesso aos pontos de ensaio, como em estradas ainda em construção. Trata-se de um ensaio dinâmico de carga com placa, e o princípio que procura reproduzir é o carregamento exercido por pneu sobre o pavimento, onde a força de impacto é gerada pela queda de uma massa sobre um sistema de amortecedores, transmitindo um impulso ao pavimento através de uma célula de carga (Benedetto et al., 2012). Esse impulso origina uma deflexão, medida por um geofone localizado no interior do cilindro do equipamento, o qual atua através de um furo no centro da placa de carga. A célula de carga e o geofone estão ligados a um dispositivo eletrônico (computador portátil), que registra automaticamente a deformação em milímetros (Machado, 2012).

Vários estudos têm demonstrado que há relações entre medidas de deflexões do LFWD com o FWD, indicando o potencial deste equipamento quanto à avaliação da integridade estrutural das camadas de pavimentos construídas por diferentes materiais. (Burhani, 2016; Nazzal et al., 2007 e Horak et al., 2008). Entretanto, o LFWD ainda é pouco difundido no Brasil, existindo carências de estudos que proponham modelos empíricos que relacionem as medidas de deflexões desse equipamento com outros tipos de deflectômetros comumente usados no país, como a Viga Benkelman e o ensaio de carga com placa.

O objetivo principal desse estudo foi avaliar o potencial de uso do LFWD para medir deflexões em pavimentos urbanos. Para isso, foram comparadas medidas de deflexões aferidas pelo LFWD, viga Benkelman e o ensaio de carga com placa em pavimentos urbanos constituídos por diferentes materiais, espessuras de revestimento asfáltico e idades de operação. A partir daí determinaram-se modelos, a partir de análise de regressão linear, que relacionaram as medidas de deflexão entre o LFWD e a VB e o ensaio de carga com placa.

\section{PROGRAMA EXPERIMENTAL}

\subsection{Vias urbanas avaliadas}

As medidas de deflexões foram efetuadas por meio de levantamentos deflectométricos com a Viga Benkelman (VB), ensaio de carga com placa (PL) e com Deflectômetro de Impacto Leve (LFWD). Estas medidas foram feitas em quatro vias urbanas localizadas na cidade de Joinville, SC, escolhidas de modo a retratar as práticas construtivas comuns à cidade.

Para a escolha das vias foram considerados: o número de camadas asfálticas (única ou dupla), o tipo de material constituinte das camadas de base e sub-base (granular ou alvenaria de paralelepípedos), a idade de operação e tipo de classificação quanto ao sistema viário (principal, coletora ou local). Foram visitadas 25 vias urbanas a fim de escolher quatro vias que fossem distintas quanto às características acima descritas. A seguir são descritas as constituições dos pavimentos das vias urbanas, subdivididas aqui nos Trechos 1, 2, 3 e 4:

- Trecho 1: o pavimento da via principal, designada como Trecho 1, foi executado sob controle tecnológico semelhante a pistas rodoviárias interurbanas. Apresenta camada de reforço de subleito em macadame seco com espessura de $30 \mathrm{~cm}$, camada de sub-base também de macadame seco de $30 \mathrm{~cm}$ de espessura, camada de base constituída por brita estabilizada granulometricamente de $15 \mathrm{~cm}$ de espessura, e revestimento de concreto 
betuminoso constituído de duas camadas, sendo $5 \mathrm{~cm}$ de Concreto Betuminoso Usinado a Quente (CBUQ) e $5 \mathrm{~cm}$ de Pré-Misturado a Quente (PMQ). 0 trecho foi liberado ao tráfego no início do ano de 2018;

- Trecho 2: este trecho localiza-se em uma via coletora que alimenta as vias principais e foi entregue ao tráfego também no início do ano de 2018. 0 pavimento é constituído por sub-base de macadame seco de $30 \mathrm{~cm}$ de espessura, base constituída por brita graduada de $15 \mathrm{~cm}$ de espessura e revestimento em CBUQ de $5 \mathrm{~cm}$ de espessura;

- Trecho 3: trata-se de uma via coletora originalmente construída em alvenaria de paralelepípedos, regularizado com camada asfáltica, sendo: $7 \mathrm{~cm}$ de espessura de PMQ coberto por 3,5 cm de CBUQ. Apesar dessa via possuir mais de 20 anos de operação em alvenaria de paralelepípedo, a sua reabilitação com revestimento em concreto asfáltico ocorreu no ano de 2017;

- Trecho 4: trata-se de uma via local constituída de um pavimento com mais de 20 anos de operação. 0 pavimento é constituído por camada de sub-base em saibro bruto de $38 \mathrm{~cm}$ de espessura, base em brita graduada de $15 \mathrm{~cm}$ de espessura e revestimento asfáltico em CBUQ de $5 \mathrm{~cm}$ de espessura.

Os levantamentos deflectométricos foram realizados entre março e junho de 2018. Cada trecho foi subdividido em dez estações de ensaios distanciadas entre si de 50 metros, com exceção do Trecho 1, que foi subdividido em oito estações de ensaio. Outras 10 estações de ensaios foram também executadas no Trecho 4 com vistas a testar o modelo empírico, a partir de regressão linear simples, entre as medidas de deflexão de diferentes equipamentos.

As medidas de deflexão foram realizadas na trilha de roda externa (bordo externo) de cada trecho, sempre em alinhamento afastado de $90 \mathrm{~cm}$ da guia do passeio (meio-fio). 0 intervalo de temperatura ambiente, registrados por ocasião dos ensaios de campo, foram de 20 a $30^{\circ} \mathrm{C}$ para o Trecho 1,18 a $26^{\circ} \mathrm{C}$ para o Trecho 2,26 a $30^{\circ} \mathrm{C}$ para o Trecho 3 e 21 a $33^{\circ} \mathrm{C}$, para o Trecho 4.

\subsection{Deflectômetros utilizados no estudo}

\subsubsection{Viga Benkelman (VB)}

No Brasil, o levantamento deflectométrico com a Viga Benkelman deve seguir os procedimentos descritos no método DNIT 133/2010 - ME. Nessa padronização, é utilizado o pneu de um caminhão como carregamento, sendo ajustado o peso de 8,2 toneladas sobre o eixo traseiro simples e de roda dupla. Sob esse eixo, entre as rodas, apoia-se uma barra de alumínio, móvel, que toca a superfície, interligando-se a uma régua fixa, por articulação. Na parte fixa é possível ler, através de um extensômetro, o deslocamento da barra de alumínio, quando o eixo (do caminhão) se afasta do ponto de início das leituras. Ocorre que a leitura observada no piso é o deslocamento vertical do mesmo durante o descarregamento.

0 método do DNIT (2010) determina que as leituras sejam feitas com o caminhão parado sobre o ponto onde devem ser realizadas as leituras (Creep Speed Rebound Deflection), procedimento este utilizado no presente estudo.

\subsubsection{Deflectômetro de Impacto Leve (LFWD)}

As especificações técnicas do LFWD utilizado no estudo pertencem ao modelo HMP-LFG4, ano de fabricação 2014 , com massa de queda de $10 \mathrm{~kg}$. A força de impacto informada pelo fabricante é de $7070 \mathrm{~N} \pm 70 \mathrm{~N}$ e duração do mesmo igual a $17 \mathrm{~ms} \pm 1,5 \mathrm{~ms}$ (milissegundos). A altura de queda da massa é de $720 \mathrm{~mm}$. 0 diâmetro da placa é de $300 \mathrm{~mm}$, com espessura de $20 \mathrm{~mm}$. 
0 peso da placa é de $15 \mathrm{~kg}$. Descreve ainda em seu manual que o intervalo de temperatura ambiente para a utilização do aparelho é de 0 - $40^{\circ} \mathrm{C}$.

Por ensaio, realizam-se seis quedas da massa, onde as três primeiras serviram de pré-compactação (assentamento da placa) e as outras três para registro da deformação. Estes três últimos registros são arquivados, sendo que o resultado do ensaio é obtido pelo valor médio das deformações. Todo o procedimento é padronização conforme a norma E2835-11 da ASTM (ASTM, 2015).

\subsubsection{Ensaio de Carga com Placa (PL)}

Neste ensaio as medidas de deflexão são obtidas mediante a aplicação de cargas sucessivas sobre uma placa circular, de raio conhecido, e na consequente medição das respectivas deformações elásticas do pavimento. O procedimento segue a norma alemã DIN 18134 (2001) que estabelece aplicação de dois ciclos de carregamento sucessivos (princípio de carga-descarga). A leitura da deformação do pavimento é registrada no relógio comparador acoplado à base da placa.

A força aplicada à placa exerce sobre a plataforma uma pressão média de $4,2 \mathrm{~kg} / \mathrm{cm}^{2}$ ao final do segundo ciclo de carregamento. Pressão essa um pouco inferior à exercida pelo pneu do caminhão no ensaio de viga, ou seja, $5,6 \mathrm{~kg} / \mathrm{cm}^{2}$. Todavia, este valor é considerado como correspondente e próximo a uma situação real de carregamento, visto que o ponto de aplicação é único. Após a estabilização da deflexão da placa determina-se, da mesma forma que para o primeiro carregamento, a deformação total da placa no final do segundo carregamento.

\subsection{Análises Estatísticas}

A estatística descritiva dos valores de deflexão medidos em cada trecho e por cada equipamento compreendeu o cálculo da média $(X)$, desvio padrão $(\sigma)$, valores máximos $\left(D_{\max }\right)$ e mínimos $\left(D_{\min }\right)$, deflexões características (DC) e coeficiente de variação (CV).

Para a determinação da relação da linearidade entre medidas de deflexão entre LFWD e VB e entre LFWD e PL foi calculado o coeficiente de correlação de Pearson $(r)$. 0 coeficiente de correlação $(r)$ é utilizado para quantificar a força da relação linear entre duas variáveis e assume valores no intervalo $-1 \leq \mathrm{r} \leq 1$, onde -1 significa uma correlação perfeita negativa entre as duas variáveis e 1 significa uma correlação perfeita positiva entre as duas variáveis. (Montgomery e Runger, 2018).

Foi utilizada a análise de regressão linear simples para modelar empiricamente as medidas de deflexão entre o LFWD e VB e entre LFWD e PL As adequações dos modelos de regressões foram avaliadas pelos coeficientes de determinação $\left(R^{2}\right)$, erros padrão e as probabilidades de significância observadas a partir dos valores P. Para julgamento comparou-se o valor P com o nível de significância adotado de 0,05.

\section{APRESENTAÇÃO E DISCUSSÃO DE RESULTADOS}

\subsection{Medidas das deflexões por trecho}

Os valores das medidas de deflexões obtidas com os equipamentos VB, PL e LFWD para cada um dos quatro trechos avaliados estão representados nas Figuras 1, 2, 3 e 4, respectivamente.

A Tabela 1 apresenta as estatísticas descritivas das medidas de deflexões obtidas, para cada trecho, e por tipo de ensaio. 


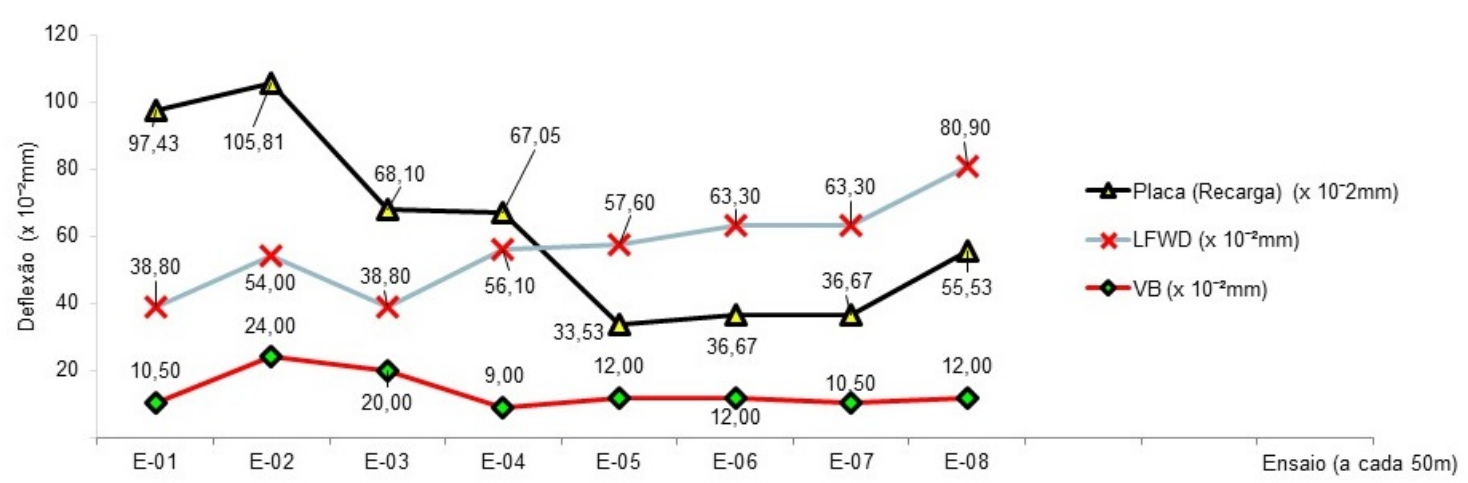

Figura 1. Deflexão (x 10-2 mm) - VB, PL e LFWD - Trecho 1

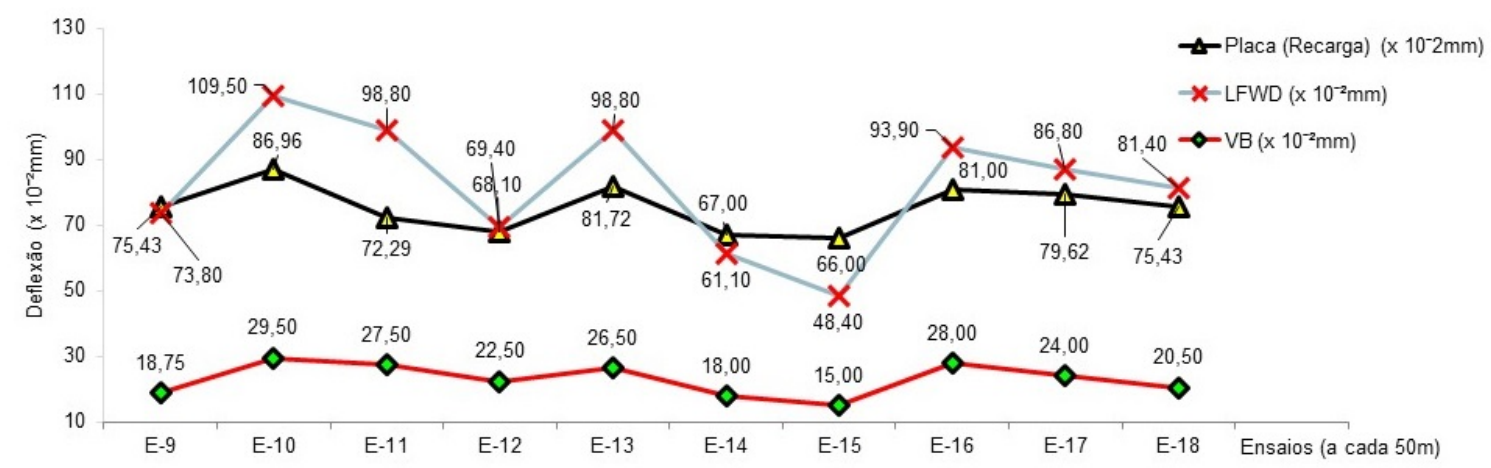

Figura 2. Deflexão (x 10-2 mm) - VB, PL e LFWD - Trecho 2

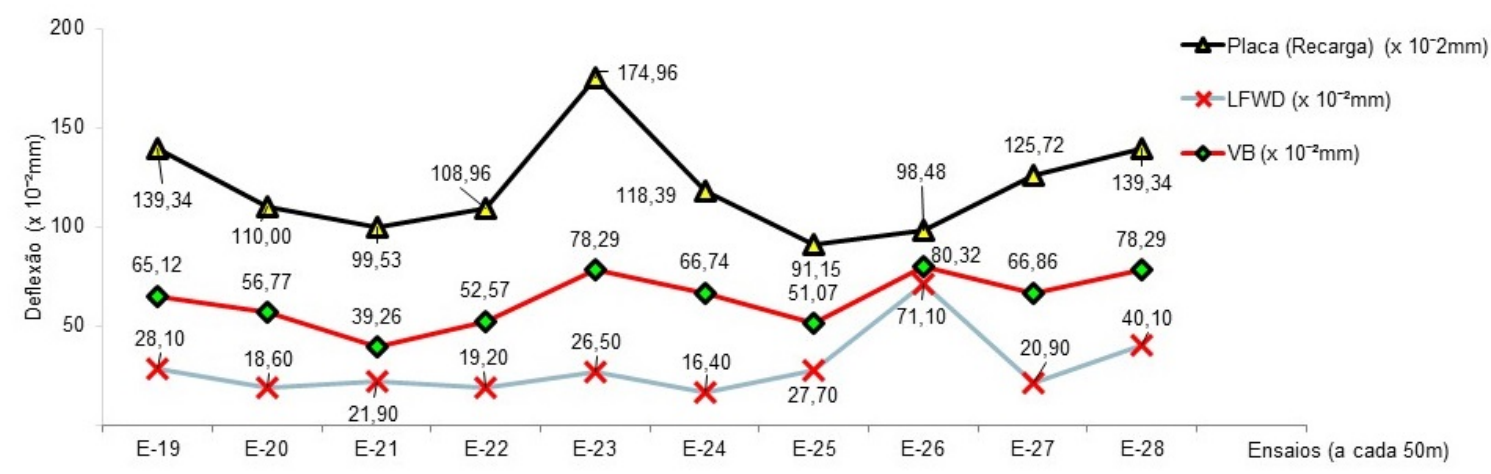

Figura 3. Deflexão (x 10-2 mm) - VB, PL e LFWD - Trecho 3

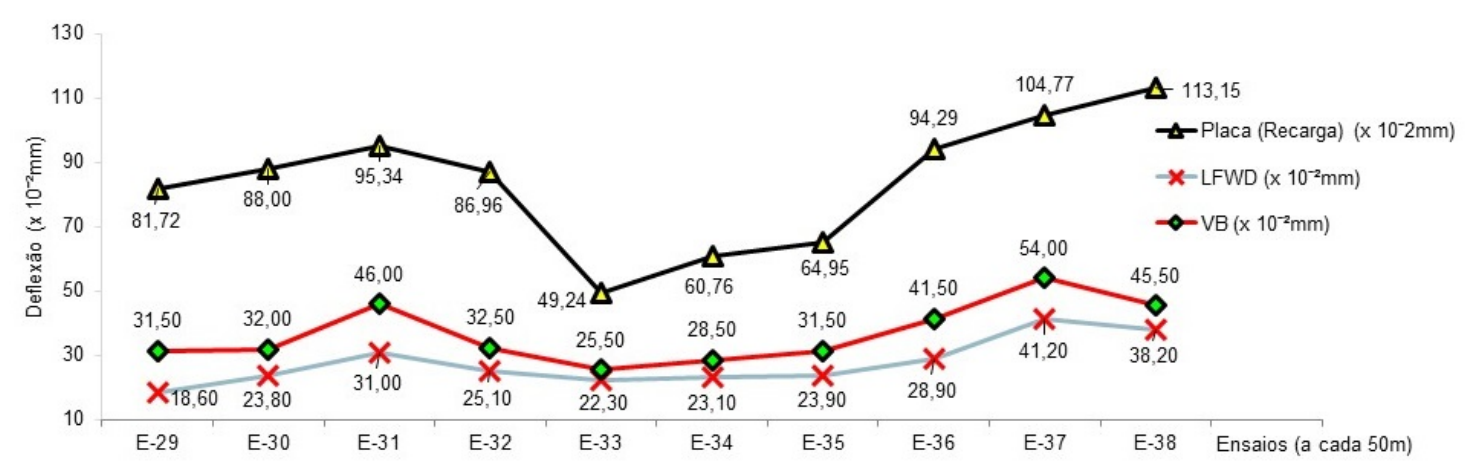

Figura 4: Deflexão (x 10-2 mm) - VB, PL e LFWD - Trecho 4 
Analisando a tabela 1 pode-se verificar que as medidas de deflexões com os três equipamentos apresentaram coeficiente de variação (CV) abaixo de 30\% para os Trechos 2 e 4, o que caracteriza maior homogeneidade entre as medidas. A norma IP-DE-P00/003 (DER-SP, 2006) específicas valores de coeficientes de variação próximos de 30\% para determinar que uma amostra seja aceita como segmento homogêneo. Deve-se ressaltar que o CV do trecho 1 do ensaio com VB e de PL, e o CV do trecho 3 do ensaio de LFWD apresentaram valores acima de 30\%, indicando que para esses trechos os valores das medidas são mais dispersos em relação à média.

Tabela 1 - Estatísticas descritivas das medidas de deflexões para cada trecho e tipo de ensaio

\begin{tabular}{|c|c|c|c|c|c|c|c|}
\hline \multicolumn{4}{|c|}{ TRECHO 1} & \multicolumn{4}{|c|}{ TRECHO 2} \\
\hline$\overline{\mathbf{x}}$ & 13,75 & 62,60 & 56,60 & $\overline{\bar{x}}$ & 23,03 & 75,35 & 82,19 \\
\hline$\sigma$ & 5,30 & 27,70 & 13,74 & $\sigma$ & 4,88 & 7,03 & 19,08 \\
\hline Dc & 19,05 & 90,30 & 70,34 & Dc & 27,90 & 82,38 & 101,27 \\
\hline Máx & 24,00 & 105,81 & 80,90 & Máx & 29,50 & 86,96 & 109,50 \\
\hline Mín & 9,00 & 33,53 & 38,80 & Mín & 15,00 & 66,00 & 48,40 \\
\hline CV\% & $39 \%$ & $44 \%$ & $24 \%$ & CV\% & $21 \%$ & $9 \%$ & $23 \%$ \\
\hline $\mathbf{N}$ & 8,00 & 8,00 & 8,00 & $\mathbf{N}$ & 10,00 & 10,00 & 10,00 \\
\hline \multicolumn{4}{|c|}{ TRECHO 3} & \multicolumn{4}{|c|}{ TRECHO 4} \\
\hline$\overline{\mathbf{x}}$ & 63,53 & 120,59 & 29,05 & $\overline{\bar{x}}$ & 36,85 & 83,92 & 27,61 \\
\hline$\sigma$ & 13,55 & 25,25 & 16,27 & $\sigma$ & 9,26 & 20,15 & 7,26 \\
\hline $\mathrm{Dc}$ & 77,08 & 145,83 & 45,32 & $\mathrm{Dc}$ & 46,11 & 104,07 & 34,87 \\
\hline Máx & 80,32 & 174,96 & 71,10 & Máx & 54,00 & 113,15 & 41,20 \\
\hline Mín & 39,26 & 91,15 & 16,40 & Mín & 25,50 & 49,24 & 18,60 \\
\hline CV\% & $21 \%$ & $21 \%$ & $56 \%$ & CV\% & $25 \%$ & $24 \%$ & $26 \%$ \\
\hline $\mathbf{N}$ & 10,00 & 10,00 & 10,00 & $\mathbf{N}$ & 10,00 & 10,00 & 10,00 \\
\hline LEC & & . & & 15 & 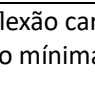 & terística; & iente \\
\hline
\end{tabular}

O Trecho 01 é um pavimento novo, ainda em fase de consolidação das camadas, gerando menor homogeneidade nas deflexões medidas, ocorrência também observada no estudo de Guimarães et al. (2015). Quanto ao elevado coeficiente de variação do Trecho 3 a partir do ensaio de LFWD, este fato ocorreu devido à falta de homogeneidade das camadas abaixo da capa asfáltica, onde existe um estrato de regularização de camada asfáltica sobre a antiga pavimentação em blocos de pedra. Essa composição induziu a uma elevada de dispersão nos resultados de deflexão. Marecos et al., (2017) também verificaram em seu estudo que as medidas com o LWFD são mais influenciadas pelas irregularidades do pavimento próximas à sua superfície.

Ainda pela tabela 3 observa-se que o intervalo de valores de deflexão obtido com a VB variaram do mínimo de 9,00 × 10-2 $\mathrm{mm}$ ao máximo de $80,32 \times 10^{-2} \mathrm{~mm}$, os valores obtidos com o

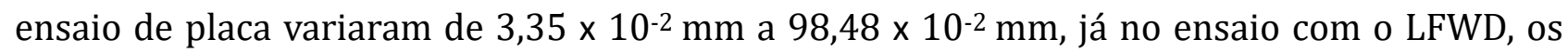
valores variaram de $16,40 \times 10^{-2} \mathrm{~mm}$ a $121,30 \times 10^{-2} \mathrm{~mm}$.

Os valores de deflexão médias obtidos considerando os quatro trechos variam de 13,75 a

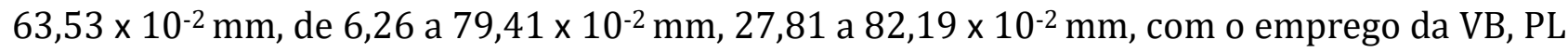
e com o LFWD, respectivamente. As deflexões características obtidas pela VB variaram de 19,05 x 10-2 mm a 77,08 $\times 10^{-2} \mathrm{~mm}$, enquanto que, para o ensaio PL foram de 9,03 $\times 10^{-2} \mathrm{~mm}$ a $89,58 \times 10^{-2} \mathrm{~mm}$, e para o LFWD variaram de $34,94 \times 10^{-2} \mathrm{~mm}$ a $101,27 \times 10^{-2} \mathrm{~mm}$.

Para a interpretação desses resultados deve-se levar em consideração que o resultado de qualquer tipo de ensaio é uma resposta direta do tipo da geometria do carregamento, magnitude da carga aplicada, da pressão de inflação do pneu no ensaio, como também do tipo de 
estrutura de pavimento ensaiado, fato também relatado nos estudos de Tholen et al., (1985) e de Moraes, (2015).

A influência do modo de carregamento sobre as deflexões pode ser melhor evidenciada quando é analisado o perfil de deflexões obtidas pelo emprego de equipamentos que utilizam modos diferentes de aplicação de carregamento, como está sendo observado no presente estudo. Nota-se, de modo geral, que as deflexões medidas com os equipamentos LFWD e PL são maiores do que aquelas medidas com os ensaios de VB.

As leituras de deflexão com a VB são influenciadas por fatores operacionais, ambientais e dependentes das condições de aplicação do carregamento. A baixa velocidade de aplicação da carga tem grande influência na resposta viscoelástica da camada asfáltica. Assim, qualquer variação no tempo de carregamento pode ser significativa para a deformação das camadas, principalmente em dias quentes, situação ocorrida nos levantamentos de campo deste estudo.

Já as diferenças de magnitudes de valores de deflexão medidas com o LFWD e o PL deve-se à profundidade de influência da tensão aplicada no ensaio de LFWD, que é aproximadamente igual a 1,5 vez o diâmetro da placa de carga usada no ensaio. Essa profundidade é menor que aquela de influência para o ensaio de carga com placa, que é aproximadamente 2 vezes o diâmetro da placa. Tais diferenças também foram observadas nos estudos de Benedetto et al., (2012), Fleming et al., (2009) e Horak et al., (2008).

\subsection{Análise de correlação e de regressão linear entre valores de deflexão da VB, LFWD e PL para cada trecho}

Na Tabela 2 são apresentados os parâmetros dos modelos de regressões lineares obtidas a partir dos valores de deflexões do LFWD com a VB e com o PL, por trecho, e os respectivos coeficientes de Pearson $(r)$, coeficientes de determinação $\left(\mathrm{R}^{2}\right)$, valores $\mathrm{P}$ e erro padrão.

Tabela 2 - Análise de correlação e regressão linear por trecho

\begin{tabular}{|c|c|c|c|c|c|c|c|c|c|c|c|c|}
\hline \multirow[t]{2}{*}{ Trecho } & \multicolumn{6}{|c|}{$\begin{array}{l}\text { Regressão Linear: } \\
D_{\mathrm{VB}}=a \cdot D_{\mathrm{LFWD}}+\mathrm{b}\end{array}$} & \multicolumn{6}{|c|}{$\begin{array}{l}\text { Regressão Linear: } \\
D_{P L}=a \cdot D_{L F W D}+b\end{array}$} \\
\hline & $a$ & $\mathrm{~b}$ & $r$ & $\mathrm{R}^{2}$ & valor $\mathrm{P}$ & Erro padrão & $\mathrm{a}$ & $b$ & $r$ & $\mathrm{R}^{2}$ & valor $\mathrm{P}$ & Erro padrão \\
\hline 1 & 0,58 & 7,09 & 0,30 & 0,28 & 0,225 & 5,17 & 2,98 & 16,94 & 0,52 & 0,12 & 0,450 & 26,50 \\
\hline 2 & 0,24 & 3,13 & 0,95 & 0,91 & 0,000 & 1,53 & 0,32 & 49,23 & 0,86 & 0,76 & 0,000 & 3,87 \\
\hline 3 & 0,47 & 49,74 & 0,57 & 0,33 & 0,108 & 12,59 & 0,20 & 126,40 & 0,13 & 0,02 & 0,742 & 27,41 \\
\hline 4 & 1,17 & 4,42 & 0,92 & 0,89 & 0,000 & 3,39 & 2,11 & 25,68 & 0,76 & 0,68 & 0,006 & 12,86 \\
\hline
\end{tabular}

Para o Trecho 1 o coeficiente de Pearson $(r)$ indica fraca correlação entre valores de deflexões entre LFWD e VB $(r=-0,30)$ e moderada correlação entre LFWD e PL $(r=-0,52)$. 0 coeficiente de determinação $\left(\mathrm{R}^{2}\right)$ das equações de regressão entre as medidas de deflexões do LFWD e VB e entre LFWD e PL são, respectivamente, iguais a 0,28 e 0,45. Os valores P de 0,225 e 0,450 são maiores que o nível de significância de 0,05. Esses valores indicam que não há evidência estatística de relação linear entre as medidas de deflexão entre LFWD e VB e entre LFWD e PL.

0 coeficiente de Pearson indicou que há forte correlação entre as medidas de deflexões entre LFWD e VB nos Trechos 2 e 4, com valores iguais a 0,95 e 0,92, respectivamente. 0 coeficiente $r$ também demonstrou que há forte correlação entre as deflexões entre LFWD e PL para esses trechos. Quanto à análise de regressão pode-se verificar que, pelos valores de $\mathrm{R}^{2}$, o modelo ajustado é adequado para ambos os trechos, tanto para as medidas de LFWD e VB e entre LFWD e PL. Tal fato também pode ser observado pelos baixos valores dos erros padrão. 
Os valores $\mathrm{P}$ indicam que há evidência estatística de relação entre as referidas medidas de deflexões.

O Trecho 3 apresentou moderada correlação entre os valores de deflexões entre LFWD e VB $(r=-0,57)$ e fraca correlação entre LFWD e PL $(r=-0,13)$. 0 coeficiente de determinação $\left(\mathrm{R}^{2}\right)$ dos modelos de regressão entre as medidas de deflexões do LFWD e VB e entre LFWD e PL são, respectivamente, iguais a 0,33 e 0,02. Os valores $P$ de 0,108 e 0,742 indicam que não há evidência estatística de relação entre as medidas de deflexão entre LFWD e VB e entre LFWD e PL, respectivamente.

Diante do exposto, pode-se observar que as melhores relações lineares entre as medidas de deflexão ocorreram em pavimentos constituídos por espessura de revestimento de $5 \mathrm{~cm}$, como nos Trechos 2 e 4. Tal ocorrência mostrou-se independente da idade de operação do pavimento, já que o Trecho 2 é uma estrutura de pavimento com cerca de 1,5 anos de operação e o Trecho 4 é um pavimento com mais de 20 anos de operação.

Não houve boa relação entre as medidas de deflexão em pavimentos constituídos por espessura de revestimento asfáltico de $10 \mathrm{~cm}$ de espessura, como os Trechos 1 e 3. 0 Trecho 1 é uma estrutura de pavimento composto de camada de revestimento asfáltico de $10 \mathrm{~cm}$ de CBUQ e de base e sub-base de materiais granulares. 0 Trecho 3 trata-se de pavimento de concreto betuminoso aplicado sobre pavimento antigo constituído de paralelepípedos, com espessura total de, aproximadamente, $25 \mathrm{~cm}$. Esta solução, geralmente executada sem projeto estrutural, é prática corrente na melhoria dos pavimentos urbanos no município de Joinville, onde o paralelepípedo é assente sobre um subleito pobre, adensado pelo tráfego de veículos ao longo dos anos.

Na Figura 5 estão representados, na forma gráfica, os valores de deflexão medidos com a VB e o LFWD nos Trechos 2 e 4 e, ainda, a reta obtida pela regressão linear, tipo $D_{V B}=a \cdot D_{L F W D}+b$, entre as referidas medidas. Foi verificado que os resíduos dos referidos modelos são independentes e normalmente distribuídos.

TRECHO 2

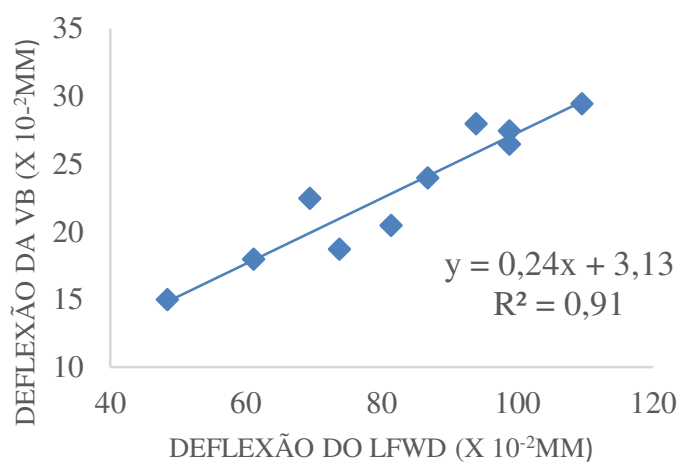

TRECHO 4

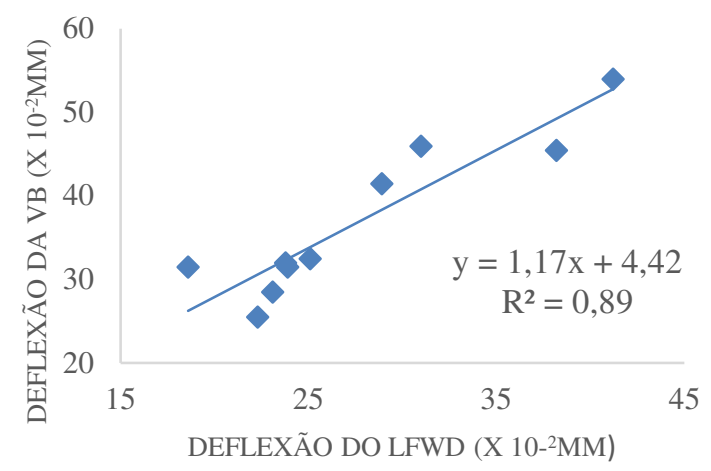

Figura 5. Regressão linear entre LFWD x VB para os Trechos 2 e 4

Na Tabela 3 são apresentados os modelos de regressão lineares e que apresentaram os maiores coeficientes de determinação $\left(\mathrm{R}^{2}\right)$ entre as medidas de deflexão para as estruturas de pavimentos avaliadas neste estudo. Observando os valores de $\mathrm{R}^{2}$ pode-se constatar que as relações entre medidas procedentes do LFWD obtidas com a VB são mais fortes do que as obtidas com o PL. 
Fica evidente que as relações lineares entre as medidas de deflexão no ponto de aplicação do carregamento são dependentes dos materiais que constituem as camadas dos pavimentos, bem como suas espessuras. As deformações elásticas em estruturas de pavimentos são influenciadas pelas diferentes condições de carregamento dos equipamentos, como exemplo: tensão aplicada, duração do pulso de carga, como também verificado nos estudos de Grasmick et al., (2015), Horak et al., (2008), Fleming et al., (2007) e Rahimzadeh et al., (2004).

Tabela 3 - Modelos de regressão lineares válidos para este estudo

\begin{tabular}{llll}
\hline Trecho & Equipamento & Modelo de Regressão e Espessura de revestimento $(\mathbf{h r})$ & $\mathbf{R}^{\mathbf{2}}$ \\
\hline 2 & Viga Benkelman & $\mathrm{D}_{\mathrm{VB}}=0,24 \cdot \mathrm{D}_{\mathrm{LFWD}}+3,13($ para $\mathrm{hr}=5 \mathrm{~cm})$ & 0,91 \\
2 & Placa com carga & $\mathrm{D}_{\mathrm{PL}}=0,32 \cdot \mathrm{D}_{\mathrm{LFWD}}+49,23($ para $\mathrm{hr}=5 \mathrm{~cm})$ & 0,76 \\
4 & Viga Benkelman & $\mathrm{D}_{\mathrm{VB}}=1,17 \cdot \mathrm{D}_{\mathrm{LFWD}}+4,42($ para $\mathrm{hr}=5 \mathrm{~cm})$ & 0,89 \\
4 & Placa com carga & $\mathrm{D}_{\mathrm{PL}}=2,11 \cdot \mathrm{D}_{\mathrm{LFWD}}+25,68($ para $\mathrm{hr}=5 \mathrm{~cm})$ & 0,68 \\
\hline
\end{tabular}

\subsection{Validação do modelo de regressão linear entre medidas de deflexão de VB e LFWD para o trecho 4}

Foram estimados os valores de deflexão da VB a partir de valores medidos com o LFWD, para as 10 estações de ensaios auxiliares e medidas no Trecho 4. Para isso foi utilizado o modelo empírico gerado a partir de regressão linear entre deflexões da VB e LFWD para o Trecho 4 e que apresentou um $\mathrm{R}^{2}$ igual a 0,89. Na Tabela 4 estão apresentadas as medidas de deflexão feitas em campo e aquelas estimadas pelo modelo empírico.

Tabela 4 - Medidas de deflexão obtidos em campo e as determinadas pelo modelo empírico para o trecho 4

\begin{tabular}{|c|c|c|c|}
\hline \multirow[t]{2}{*}{ ENSAIOS AUXILIARES TRECHO 4} & \multicolumn{2}{|c|}{$\begin{array}{c}\text { Deflexão }\left(\times 10^{-2} \mathrm{~mm}\right) \text { - Levantamento de } \\
\text { Campo }\end{array}$} & \multirow{2}{*}{$\begin{array}{c}\text { Deflexão }\left(\times 10^{-2} \mathrm{~mm}\right) \text { obtida pela regressão } \\
\text { linear } \\
D_{\mathrm{VB}}=1,17 \cdot \mathrm{D}_{\mathrm{LFWD}}+4,42\end{array}$} \\
\hline & VB & LFWD & \\
\hline E-29a & 28 & 48 & 61 \\
\hline $\mathrm{E}-30 \mathrm{a}$ & 30 & 27 & 36 \\
\hline $\mathrm{E}-31 \mathrm{a}$ & 28 & 16 & 23 \\
\hline$E-32 a$ & 28 & 20 & 28 \\
\hline $\mathrm{E}-33 \mathrm{a}$ & 27 & 25 & 34 \\
\hline $\mathrm{E}-34 \mathrm{a}$ & 34 & 20 & 28 \\
\hline$E-35 a$ & 32 & 21 & 29 \\
\hline E-36a & 33 & 24 & 32 \\
\hline E-37a & 36 & 19 & 27 \\
\hline $\mathrm{E}-38 \mathrm{a}$ & 31 & 15 & 23 \\
\hline Média $\left(\times 10^{-2} \mathrm{~mm}\right)$ & 30,45 & & 32,00 \\
\hline Desvio $\left(\times 10^{-2} \mathrm{~mm}\right)$ & 3,11 & & 10,90 \\
\hline Característica $\left(\times 10^{-2} \mathrm{~mm}\right)$ & 33,56 & & 42,90 \\
\hline Máximo $\left(\times 10^{-2} \mathrm{~mm}\right)$ & 35,50 & & 60,56 \\
\hline Mínimo $\left(\times 10^{-2} \mathrm{~mm}\right)$ & 26,50 & & 22,51 \\
\hline Coeficiente de Variação (\%) & $10,22 \%$ & & $34,07 \%$ \\
\hline $\mathrm{N}^{\circ}$ de estações & 10,00 & & 10,00 \\
\hline
\end{tabular}

Pela tabela 4 verifica-se que, com exceção do ensaio auxiliar E-29a, os valores de deflexão de VB obtidos pelo modelo empírico são bastante próximos aos aferidos com a VB, para os mesmos locais de ensaios em campo.

A plotagem dos valores de deflexão para a VB, medidos em campo, bem como os valores de deflexão calculados a partir do modelo estão apresentados na Figura 6. Foi considerado o intervalo do desvio padrão de $[-\sigma,+\sigma]$, obtido estatisticamente, para comprovação de que somente o ponto de ensaio E-29a ficou fora do intervalo. 


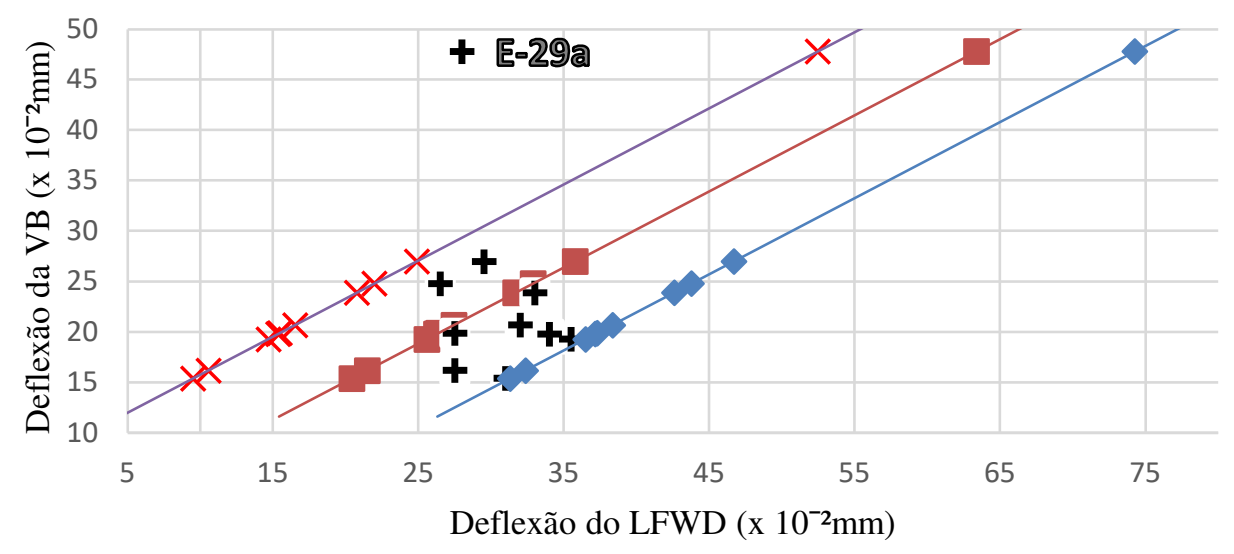

Deflexão VB Calc. + Deflexão VB medida • VB Calc + Desv.P XVB Calc - Desv.P

Figura 6. Plotagem dos valores medidos em campo e obtidos pela equação de regressão linear do tipo $D_{V B}=a \cdot D_{L F W D}+$ b para o Trecho 4

\section{CONCLUSÕES}

Neste estudo constatou-se que o LFWD apresenta potencial de uso para medir deformações elásticas em pavimentos urbanos, entretanto, algumas considerações devem ser feitas:

- não houve boa correlação e relação linear entre as medidas de deflexão entre LFWD e VB e entre LFWD e PL em estruturas de pavimentos compostas por camadas de revestimento asfáltico duplo de $10 \mathrm{~cm}$ ( $5 \mathrm{~cm}$ de CBUQ e $5 \mathrm{~cm}$ de PMQ), como também em estruturas de revestimento asfáltico sobre paralelepípedos;

- houve boa correlação e relação linear entre as medidas de deflexão obtidas entre LFWD e VB e entre LFWD e PL em estruturas de pavimentos compostas por revestimento em CBUQ mais delgada, de espessura de $5 \mathrm{~cm}$, sobre base granular.

Tal fato corrobora com os estudos de Rahimzadeh et al. (2004) e de Fleming et al. (2007) que afirmaram que a relação entre medidas de deflexão de diferentes equipamentos são dependentes do tipo de material constituinte das camadas do pavimento, além de suas espessuras.

A relação linear mais forte ocorreu entre as medidas de deflexão entre LFWD e a VB, sem levar em consideração a idade de operação do pavimento e seu tipo de classificação no sistema viário. Porém, é notório que não se pode propor um modelo empírico que seja único para pavimentos que apresentam composição de estruturas distintas.

Nesse sentido, sugere-se que, para uma determinada via em avaliação, sejam executadas ilhas de investigação em pavimentos que apresentam a mesma estrutura de modo a se definir um modelo empírico, por regressão linear, entre dados de ensaios deflectométricos com a VB e o LFWD. Com o modelo gerado, determinar-se-á, com a execução de ensaios com o LFWD, as deformações elásticas ao longo do perfil longitudinal da via em questão, como uma alternativa de rápida execução e sem grande interferência no tráfego da via.

Deve-se dar preferência ao uso de equipamentos automatizados como o LFWD pois, este tipo de equipamentos elimina a possibilidade de ocorrência de erros usualmente associados à manipulação inadequada dos equipamentos manuais, bem como de erros grosseiros de leitura e registro das medidas de deflexão.

Para novos estudos, torna-se importante destacar que a avaliação estrutural de pavimentos por deflexão, por si só, não explica o desempenho da estrutura. Portanto, esta prática deve estar 
associada a resultados de ensaios de laboratório, como a determinação do módulo resiliente dos materiais aplicados na construção do pavimento, verificação de umidade (drenagem existente) e do grau de compactação do subleito.

\section{REFERÊNCIAS}

Albernaz, C. A. V. (1997) Método simplificado de retroanálise de módulos de resiliência de pavimentos flexíveis a partir da bacia de deflexão. Dissertação (mestrado), COPPE-UFRJ, Rio de Janeiro.

AASHTO (1993) AASHTO Guide for Design of Pavement Structures. Vol. 1, American Association of State Highway, and Transportation Officials, Washington, D.C.

ASTM (2015) E2835-11 Standard Test Method for Measuring Deflections using a Portable Impulse Plate Load Test Device, American Society for Testing and Materials.

Benedetto, A.; F. Tosti e L. Di Domenico (2012) Elliptic model for prediction of deflections induced by a Light Falling Weight Deflectometer. Journal of Terramechanics, v. 49, n. 1, p. 1-12. DOI:10.1016/j.jterra.2011.10.003

Bernucci, L. B.; L. M. G. Motta; J.A.P. Ceratti e J.B. Soares (2007) Pavimentação Asfáltica: Formação Básica para Engenheiros. PETROBRAS/ABEDA, Rio de Janeiro.

Burhani, A. (2016) Correlation Study on the Falling Weight Deflectometer and Light Weight Deflectometer for the Local Pavement Systems. Master of Science. Faculty of the Russ College of Engineering and Technology of Ohio University, Ohio, EUA.

DER-SP (2006) IP-DE-P00/003 - Avaliação funcional e estrutural de pavimento. Instrução de Projeto. Departamento de Estradas e Rodagem, São Paulo, SP.

DIN (2001) 18134 Determining the deformation and strenght characteristics of soil by plate loading test. Deutsches Institut für Normung. Germany.

DNIT (2010) 133/2010 - Pavimentação asfáltica - Delineamento da linha de influência longitudinal da bacia de deformação por intermédio da Viga Benkelman - Método de ensaio. Diretoria de Planejamento e Pesquisa, Departamento Nacional de Infraestrutura de Transportes, Rio de Janeiro, RJ.

Elhakim, A. F.; K. Elbaz e M. Amer (2014) The use of light weight deflectometer for in situ evaluation of sand degree of compaction. HBRC Journal, v. 10, n. 3, p. 298-307. DOI:10.1016/j.hbrcj.2013.12.003

Fleming, P. R.; M. W. Frost e J. P. Lambert (2007) Review of Lightweight Deflectometer for Routine In Situ Assessment of Pavement Material Stiffness. In Transportation Research Record: Journal of the Transportation Research Board, $N^{\circ} 2004$, pp. 80-87. DOI:10.3141/2004-09

Fleming, P. R.; M.W. Frost e J. P. Lambert (2009) Lightweight Deflectometers for quality assurance in road construction. Bearing Capacity of Roads, Railways and Airfields: Proceedings of the 8th International Conference (BCR2A'09), University of Illinois at Urbana - Champaign, Champaign, Illinois, USA, pp. 809-818.

Francisco, A. (2012) Comportamento estrutural de pavimentos rodoviários flexíveis. Tese (Doutorado). Instituto Politécnico de Bragança, Escola Superior de Tecnologia e Gestão.

Garcia, J. A. R.; M. Castro (2011) Analysis of the temperature influence on flexible pavement deflection. Construction and Building Materials, n. 25, p. 3530-3539. DOI: 10.1016/j.conbuildamat.2011.03.046

Grasmick, J. G.; M A. Mooney; C. T. Senseney; R. W. Surdahl e M. Voth (2015) Comparison of Multiple Sensor Deflection Data From Lightweight and Falling Weight Deflectometer Tests on Layered Soil, Geotechnical Testing Journal, Vol. 38, No. 6, pp. 851-863. DOI:10.1520/GTJ20140172

Guimarães, O.R.; L. R. Rezende e M. T. Rocha (2015) Uso da Viga Benkelman para monitoramento de rodovias. Anais do 44 Reunião Anual de Pavimentação, RAPv, Foz do Iguaçu, PR.

Horak, E.; J. Maina; D. Guiamba e A. Hartman (2008) Correlation Study with lightweight Deflectometer in South Africa. Proc. 27th Southern African Transport Conference, Pretoria, South Africa.

Lopes, J. (2010) Contributo para o estudo da utilização do deflectómetro de impacto ligeiro. Dissertação (Mestrado). Instituto Superior Técnico, Lisboa.

Machado, A. B. A. S. (2012) Contribuição para a avaliação estrutural de infraestruturas rodoviárias por métodos não destrutivos. Dissertação (Mestrado). Faculdade de Ciências e Tecnologia. Universidade Nova de Lisboa.

Marecos, V.; M. Solla; S. Fontul e V. Antunes (2017) Assessing the pavement subgrade by combining different non-destructive methods. Construction and Building Materials, n. 135, p. 76-85. DOI: 10.1016/j.conbuildamat.2017.01.003

Montegomery, D. C.; C. G. Runger (2018) Estatística Aplicada e Probabilidade para Engenheiros. Rio de Janeiro, RJ. LTC - Livros Técnicos e Científicos Editora. 6⿳a ed., 627 p.

Moraes, C. G. (2015) Análise de bacias deflectométricas obtidas por 4 equipamentos do tipo Falling Weight Deflectometer (FWD). Dissertação (Mestrado). Universidade Federal do Rio de Janeiro.

Nazzal, M. D. (2003) Field evaluation of in-situ test technology for QC/QA during construction of pavement layers and embankments. Master's Theses. Louisiana State University.

Nazzal, M. D.; M. Y. Abu-Farsakh; K. Alshibli e L. Mohammad (2007) Evaluating the Light Falling Weight Deflectometer Device for In Situ Measurement of Elastic Modulus of Pavement Layers. Transportation Research Record, n. 2016, pp. 13-22. DOI:10.3141/2016-02

Rahimzadeh, H. B. e B. J. Martyn (2004) Performance Testing of Unbound Materials within the Pavement Foundation. Proc. 6th Unbound Aggregates in Roads Symposium (UNBAR6), Nottingham, UK 
Tholen, O.; J. Sharma e R. L. Terrel (1985) Comparison of Falling Weight Deflectometer with other Deflection Testing Devices. Transportation Research Record 1007. Transportation Research Board, Washington, D.C., pp 20 - 26. 